\title{
Hipertensión renovascular: actualización
}

\author{
Ricardo Silvariño ${ }^{1 *}$, José Boggia' ${ }^{1}$ Sofía San-Román ${ }^{1}$, Cecilia Baccino ${ }^{1}$, Alejandro Crisci², \\ Marcelo Langleib ${ }^{2}$ y Óscar Noboa ${ }^{1}$ \\ ${ }^{1}$ Centro de Nefrología; ${ }^{2}$ Cátedra de Imagenología. Hospital de Clínicas, Facultad de Medicina, Universidad de la República, Montevideo, Uruguay
}

\section{Resumen}

La hipertensión renovascular (HTRV) es una causa frecuente y potencialmente corregible de hipertensión arterial (HTA) secundaria. Su incidencia varía en función del contexto clínico. Se encuentra estenosis de la arteria renal (EAR) en $1 \%$ de los pacientes con HTA leve y hasta en $10 \%$ a $45 \%$ de los pacientes con HTA grave o evolución acelerada. Las causas más frecuentes de EAR son la enfermedad vascular aterosclerótica y la displasia fibromuscular (DFM), que se identifican con una relación 9:1 en favor de la primera. Otras enfermedades vasculares mucho menos frecuentes pueden ser causa de EAR. La HTRV aparece por lo regular en un contexto de elevada comorbilidad cardiovascular y coexiste con enfermedad coronaria (10\%-14\%), enfermedad arterial periférica (15\%-25\%), cerebrovascular (10\%), aórtica (25\%-35\%) y enfermedad renal crónica (ERC) avanzada (35\%), entre otras. Este escenario en el que ocurre la HTRV representa dificultades diagnósticas y terapéuticas. Algunas de las preguntas que subyacen al tratamiento de estos pacientes son: ¿debe buscarse EAR en un paciente determinado?, ¿cuál es la forma de estudio más adecuada?, ¿debe instituirse tratamiento médico o intervencionista en un paciente específico? Esta revisión intenta responder algunas de estas preguntas con un enfoque predominantemente clínico y basado en la información actualizada. Dado el papel preponderante que tiene la enfermedad aterosclerótica por su frecuencia, la atención se enfoca en ella. Mucho más que en otras alteraciones, el abordaje de la HTRV debe ser grupal, con intervención de las diferentes disciplinas para tomar decisiones.

Palabras clave: Hipertensión renovascular. Hipertensión arterial secundaria. Estenosis de la arteria renal.

\section{Renovascular hypertension. State of the art}

\section{Abstract}

Renovascular hypertension (RVHT) is a frequent and potentially correctable cause of secondary arterial hypertension (HT). Its incidence varies depending on the clinical setting. Renal artery stenosis (RAS) is found in $1 \%$ of patients with mild HT and in up to $10-45 \%$ in patients with severe HT or with accelerated evolution. The most frequent causes of RAS are atherosclerotic vascular disease and fibromuscular dysplasia (FMD), which are found in a ratio 9:1 in favor of the atherosclerotic disease. RVHT usually occurs in a context of high cardiovascular comorbidity, coexisting with coronary heart disease (10-14\%), peripheral arterial disease (15-25\%), cerebrovascular (10\%), aortic (25-35\%) and advanced chronic kidney disease (CKD) (35\%), among others. Diagnostic and therapeutic difficulties arise in different clinical scenarios. Some of the questions underlying the approach of these patients are: should I look for RAS in a specific patient? What is the most appropriate form

\section{Correspondencia:}

${ }^{\star}$ Ricardo Silvariño

E-mail: rsilvarino@gmail.com;

Fecha de recepción: 13-07-2020

Fecha de aceptación: 22-10-2020

DOI: $10.24875 /$ NEFRO.20000031
Disponible en internet: 17-12-2020 Nefro Latinoam. 2020;17:125-138 www.nefrologialatinoamericana.com rsilvarino@hc.edu.uy 2444-9032/@ 2020 Sociedad Latinoamericana de Nefrología e Hipertensión. Publicado por Permanyer. Este es un artículo open access bajo la licencia CC BY-NC-ND (http://creativecommons.org/licenses/by-nc-nd/4.0/). 
Nefro Latinoam. 2020;17

of study? Should I carry out medical and I or interventional treatment in a specific patient? We review these questions from a clinical approach and based on the "state of the art". Given the preponderant role of atherosclerotic disease due to its frequency, the emphasis will be placed on it. Much more than other pathologies, the RVHT approach must be in a team, integrating the different disciplines in decision-making.

Key words: Renovascular hypertension. Secondary arterial hypertension. Renal artery stenosis.

\section{Introducción}

La hipertensión renovascular (HTRV) es todavía la causa más prevalente, y potencialmente reversible, de hipertensión arterial (HTA) secundaria'. La estenosis de la arteria renal (EAR) significativa de origen aterosclerótico es la causa más frecuente y ocurre con regularidad en el contexto de pacientes con factores de riesgo vascular ${ }^{2}$, en quienes la HTA coexiste, aun cuando la EAR no sea la causa primaria de $\mathrm{HTA}^{3}$. Este vínculo entre la presencia de HTA, factores de riesgo vascular, ateromatosis y EAR da lugar a dificultades diagnósticas y sobre todo terapéuticas. Las disyuntivas vinculadas con el tratamiento intervencionista son en particular complejas en la EAR aterosclerótica, en la cual la evidencia disponible hasta el momento se inclina por una estricta selección del grupo de pacientes que podrían beneficiarse de él junto con el tratamiento médico ${ }^{1,4}$. Se revisa de forma progresiva la definición y terminología relacionadas con HTRV, fisiopatología, etiología, metodología de estudio y tratamiento. En función de las causas más prevalentes, este trabajo describe la EAR de origen aterosclerótico y la displasia fibromuscular.

\section{Definiciones y terminología actual}

Hipertensión renovascular (HTRV) es el término empleado para definir el desarrollo de hipertensión arterial (HTA), secundaria a la disminución significativa del calibre de la arteria renal (estenosis de la arteria renal) principal unilateral o bilateral ${ }^{2}$. La estenosis de la arteria renal (EAR) señala la disminución significativa (casi siempre $>60 \%-70 \%$ ) del calibre de la arteria renal ${ }^{2}$. La enfermedad renal crónica (ERC) se refiere a un conjunto heterogéneo de trastornos que afectan la estructura o función del riñón; se define por la presencia de alteraciones estructurales (albuminuria, alteraciones del sedimento urinario, alteraciones histológicas en la biopsia renal, alteraciones anatómicas en técnicas de imagen) o funcionales (descenso del filtrado glomerular, disfunción tubular, entre otras), mantenidas durante al menos tres meses y con implicaciones para la salud ${ }^{5}$. Nefropatía isquémica es el término empleado para

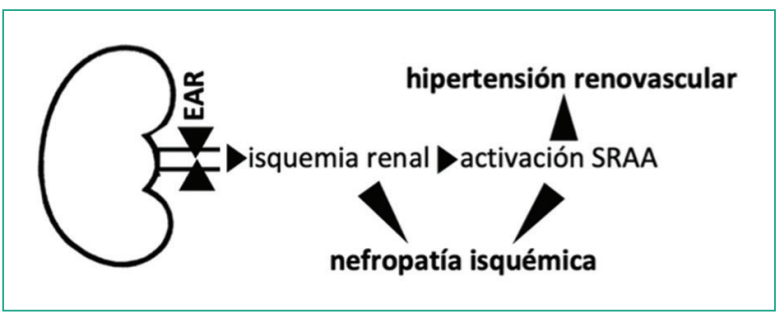

Figura 1. La EAR se refiere a la disminución significativa (casi siempre $>60 \%-70 \%$ ) del calibre de la arteria renal. HTRV es el término empleado para definir el desarrollo de la hipertensión arterial secundaria a EAR. Nefropatía isquémica describe el desarrollo de ERC consecutiva a la isquemia mantenida del parénquima renal. EAR: estenosis de la arteria renal; HTRV: hipertensión renovascular; SRAA: sistema renina-angiotensina-aldosterona.

describir el desarrollo de ERC secundaria a la isquemia mantenida del parénquima renal ${ }^{6}$ (Fig. 1).

La EAR es efecto de HTRV y nefropatía isquémica. En este capítulo se revisa la fisiopatología, etiología, epidemiología y abordajes clínico y terapéutico de la HTRV como manifestación de EAR. No se describen en forma detallada otras manifestaciones clínicas 0 analíticas de la EAR.

\section{Fisiopatología}

Goldblatt, et al. describieron el primer modelo animal (perro) generado para estudiar el desarrollo de HTA en 1934 ${ }^{7}$. Consistía en la constricción parcial de una o ambas arterias renales a través de una grapa metálica. En función de la presencia de uno o los dos riñones, o de la utilización de una o dos grapas metálicas, se integraron tres variaciones sobre el modelo de HTRV: 2 riñones +1 grapa (2R1G), 1 riñón + 1 grapa (1R1G) y 2 riñones +2 grapas (2R2G).

En la experiencia ${ }^{7}$ con el modelo $2 \mathrm{R} 1 \mathrm{G}$ se produjo HTA y se observó que el riñón contralateral (sin grapa) incrementaba la excreción de sodio (natriuria) como respuesta funcional a la HTA. Este modelo se identifica como el mecanismo fundamental en la HTRV. De manera progresiva se identificó el papel del sistema renina-angiotensina-aldosterona (SRAA) 


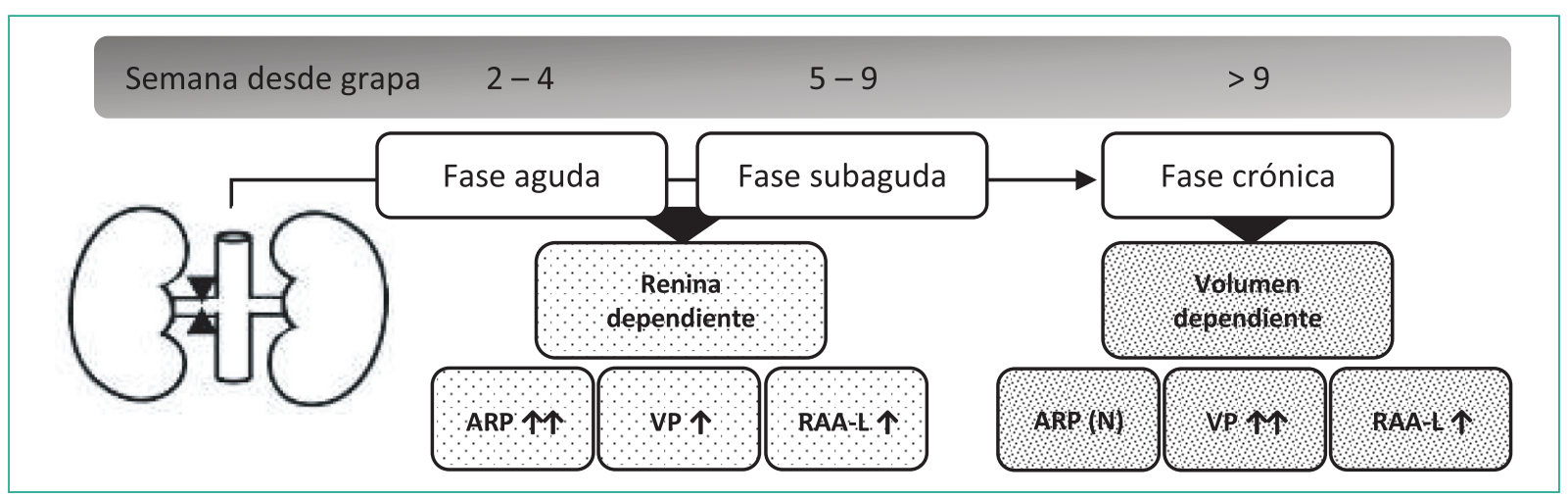

Figura 2. Alteraciones fisiopatológicas evolutivas en el modelo 2R1G (Adaptado de Xin Z, et al. ${ }^{10}$ ). ARP: actividad de renina plasmática; RAA-L: sistema renina-angiotensina-aldosterona local; VP: volumen plasmático.

en la base neurohumoral de este mecanismo. Los investigadores argentinos del Laboratorio de B. Houssay (Menéndez, Fasciolo y Taquini) descubren la "hipertensina" que luego se denominaría "angiotensina" tras integrar el descubrimiento de Page, et al. un año después ${ }^{8}$. En el modelo 2R1G se documentaron valores muy elevados de renina plasmática circulante y aldosterona, sobre todo en la fase inicial de la estenosis con la grapa. Sin embargo, si este mecanismo de lesión se prolonga en el tiempo aparecen mecanismos de adaptación. Se han descrito tres fases temporales en la respuesta fisiológica al modelo 2R1G:

- Una fase aguda (2-4 semanas luego de la aplicación de la grapa), una fase subaguda (5-9 semanas después de la aplicación de la grapa). En estas fases, la HTA se caracteriza por cursar con cifras elevadas de renina plasmática.

- En la fase crónica (más de 9 semanas posteriores a la aplicación de la grapa), las concentraciones plasmáticas de renina regresan a su valor normal. La HTA se mantiene a costa de una expansión del volumen plasmático (HTA dependiente del volumen), la acción de SRAA locales (en el riñón engrapado) o de ambos mecanismos en conjunto ${ }^{9}$ (Fig. 2) ${ }^{10}$.

En el modelo 1R1G se produjo HTA, pero la ausencia de riñón contralateral (removido de manera previa) no permitió una excreción de sodio y agua compensatorios. Esto causó retención hidrosalina y por tanto el mantenimiento de HTA dependiente de volumen. La ausencia de incremento compensatorio de la natriuresis del riñón contralateral diferenció a este modelo del primero ${ }^{7}$. En el modelo 2R2G, los mecanismos fisiopatológicos son similares a los del 1R1G.

\section{Etiología}

La enfermedad vascular aterosclerótica es la causa más frecuente de HTRV. Se trata de un espectro de cambios de origen aterosclerótico de las arterias tributarias del riñón, que en términos morfológicos se expresa como estenosis focal de una 0 más arterias renales (EAR), isquemia con atrofia renal u oclusión total de la circulación arterial. No hay consenso que defina en qué momento una estenosis es significativa $y$, a pesar de ello, la mayor parte de los estudios que incluyen a pacientes con HTRV registra oclusiones de $50 \%, 60 \%$ o $75 \%$ del diámetro arterial ${ }^{2,11,12}$. Los estudios en pacientes con EAR unilateral señalan que sólo cuando la estenosis alcanza una proporción de 70\% a $80 \%$ del diámetro vascular se incrementa la secreción de renina ${ }^{13}$. Sin embargo, hay que considerar que la aterosclerosis es una enfermedad sistémica. Cualquiera que sea el grado de EAR, el paciente con enfermedad vascular muestra con frecuencia algún grado de estenosis en la arteria contralateral, ateromatosis difusa de la aorta y las arterias intrarrenales, y enfermedad aterosclerótica de pequeño vaso. Es por ello que algunos autores consideran que es suficiente para definir EAR significativa la presencia de estenosis $>50 \%$ vinculada con el daño del parénquima renal, alteración neurohormonal (activación del SRAA) o cambios crónicos de la estructura o función cardiaca sin otra clara causa que los explique ${ }^{14}$.

La prevalencia de la enfermedad vascular renal aterosclerótica es difícil de determinar. Los estudios al respecto registran prevalencias diferentes en función de los subgrupos de población estudiados. En la población general asintomática se diagnostica habitualmente en el contexto de otro estudio vascular 
(angiotomografía, coronariografía, arteriografía) ${ }^{14}$. En un estudio en los que se valoró con Doppler de arterias renales a 834 pacientes mayores de 65 años asintomáticos, se encontró EAR (> 60\%) en $6.8 \%$ de los

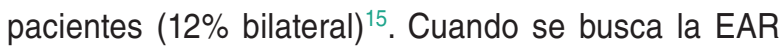
en poblaciones con factores de riesgo o enfermedad vascular establecida, la prevalencia aumenta (Tabla 1).

Los factores de riesgo para desarrollar EAR aterosclerótica son los mismos observados en el desarrollo de la aterosclerosis en otros territorios. En un estudio, Kalra, et al. ${ }^{16}$ valoraron el peso de la presencia de enfermedad cardiovascular previa y los factores de riesgo vascular sobre la prevalencia de EAR aterosclerótica. En la Tabla 2 se resumen los resultados.

La progresión de la EAR aterosclerótica es un hecho frecuente. En un estudio prospectivo, Zierler, et al. ${ }^{17}$ realizaron seguimiento mediante Doppler de arterias renales en 76 pacientes, en los que se halló alguna anormalidad arterial (distinta de EAR) durante un estudio inicial, y encontraron que la incidencia acumulativa de progresión desde una estenosis $<60 \%$ a una $>60 \%$ fue de $30 \%$ a un año, $44 \%$ a dos años y $48 \%$ a tres años. Al valorar todas las categorías de lesiones, concluyeron que existe una progresión promedio de la lesión de 7\% anual. Caps, et al. ${ }^{18}$ vigilaron (33 meses de seguimiento) con Doppler seriado de arterias renales a 170 pacientes con EAR conocida e identificaron que la incidencia acumulativa de progresión fue de $35 \%$ a tres años y de $51 \%$ a cinco años.

La estenosis de una arteria renal accesoria es otro aspecto a considerar. Se han descrito arterias accesorias hasta en $25 \%$ a $50 \%$ de la población general en estudios de necropsia ${ }^{19}$, pero su contribución al desarrollo de HTRV es controvertido. Mientras algunos estudios reducen al mínimo su participación en el desarrollo de HTRV ${ }^{20}$, otros informes demuestran el peso que esta variante arterial anatómica puede tener en el desarrollo de la hipertensión arterial ${ }^{21,22}$. Hay consenso en que deben buscarse estenosis en arterias accesorias en pacientes en los que se estudia un posible origen renovascular de la hipertensión arterial ${ }^{23,24}$.

La HTRV es una de las principales manifestaciones clínicas de EAR aterosclerótica. Otras manifestaciones clínicas relacionadas con EAR se resumen en la figura 3.

La displasia fibromuscular (DFM) es una enfermedad idiopática, segmentaria, no aterosclerótica ni inflamatoria, que afecta la pared muscular de las arterias, lo que provoca estenosis en los vasos de pequeño y mediano calibre ${ }^{25}$. Desde el punto de vista angiográfico
Tabla 1. Prevalencia de estenosis de arteria renal en pacientes con factores de riesgo cardiovascular 0 enfermedad vascular establecida en otros territorios

\begin{tabular}{|l|c|c|}
\hline Grupo de riesgo & $\begin{array}{c}\text { Estenosis de AR } \\
\text { (unilateral o } \\
\text { bilateral) (\%) }\end{array}$ & $\begin{array}{c}\text { Estenosis de AR } \\
\text { bilateral (\%) }\end{array}$ \\
\hline $\begin{array}{l}\text { CACG por síndrome } \\
\text { coronario } \\
\text { Sospecha de HTRV }\end{array}$ & 10.5 & 1.7 \\
\hline $\begin{array}{l}\text { Síndrome coronario + } \\
\text { sospecha de HTRV }\end{array}$ & 14.1 & 4.6 \\
\hline $\begin{array}{l}\text { Estudio con CACG en } \\
\text { población con HTA }\end{array}$ & 17.8 & 4.3 \\
\hline $\begin{array}{l}\text { Diabetes mellitus con HTA } \\
\text { Enfermedad vascular } \\
\text { periférica }\end{array}$ & 20 & 6 \\
\hline $\begin{array}{l}\text { Aneurisma de aorta } \\
\text { abdominal }\end{array}$ & 25.3 & 13.4 \\
\hline $\begin{array}{l}\text { Enfermedad renal crónica } \\
\text { extrema }\end{array}$ & 43.1 & 12.8 \\
\hline $\begin{array}{l}\text { Insuficiencia cardíaca } \\
\text { congestiva }\end{array}$ & 54.1 & 12.2 \\
\hline AR & & 23.7 \\
\hline
\end{tabular}

AR: arteria renal; CACG: cineangiocoronariografía; HTA: hipertensión arterial; HTRV: hipertensión renovascular.

Adaptado de De Mast, et al..$^{48}$

se presenta con dos patrones radiológicos: DFM focal, caracterizada por una estenosis focal que puede ocurrir en cualquier parte de la arteria, y DFM multifocal, cuando alterna áreas de estenosis con áreas de dilatación ("cadena de cuentas"), y se observa por lo regular en las porciones media o distal de las arterias ${ }^{25}$ (Fig. 4). Además de la clasificación radiológica, que es la de mayor interés práctico, existe una clasificación histológica que divide la DFM en función del área de la arteria más afectada. En la gran mayoría de los casos se afecta la media arterial $(85 \%-100 \%)$. En raras ocasiones puede afectarse la íntima $(<10 \%)$ o la adventicia $(<1 \%)$ de la arteria ${ }^{26}$. En la tabla 3 se presentan las características histológicas de los diferentes tipos de lesiones.

La DFM puede dañar cualquier territorio arterial. En la tabla 4 se presenta la distribución de acuerdo con el lecho vascular afectado. Los registros más importantes de DFM documentan edad promedio al diagnóstico en la quinta y sexta décadas de la vida, con predominio de mujeres de raza blanca. En América Latina, el Registro Nacional Argentino de Displasia Fibromuscular (SAHARA-DF) inició en 2015, y en 2019 incluía a 49 pacientes con diagnóstico confirmado, de los cuales 44 (89.7\%) 
Tabla 2. Factores de riesgo vinculados con el desarrollo de EAR

\begin{tabular}{|l|c|c|c|c|}
\hline & No EAR $(\mathbf{n}=\mathbf{1 , 0 8 5 , 2 5 0 )}$ & EAR $(\mathbf{n}=5,875)$ & EAR HR $($ IC95 \%) & p \\
\hline Lesión renal aguda (\%) & 0.8 & 10.3 & $1.59(1.43-1.77)$ & $<0.0001$ \\
\hline Enfermedad renal crónica & 2.3 & 24.6 & $4.61(4.27-4.98)$ & $<0.0001$ \\
\hline Hipertensión arterial & 53.4 & 90.8 & $4.31(3.93-4.73)$ & $<0.0001$ \\
\hline Diabetes mellitus & 17.9 & 32.5 & $0.89(0.84-2.61)$ & 0.0001 \\
\hline Enfermedad coronaria & 24.9 & 66.8 & $2.45(2.3-2.61)$ & $<0.0001$ \\
\hline Insuficiencia cardiaca congestiva & 13.6 & 37.6 & $1.01(0.94-1.07)$ & 0.9 \\
\hline ACV/AIT & 12 & 36.9 & $1.58(1.49-1.67)$ & $<0.0001$ \\
\hline Enfermedad vascular periférica & 12.7 & 56 & $3.96(3.74-4.2)$ & $<0.0001$ \\
\hline Isquemia mesentérica & 0.2 & 1.9 & $2.38(1.93-2.93)$ & $<0.0001$ \\
\hline Aneurisma de aorta abdominal & 0.5 & 6.4 & $3.38(3.0-2.81)$ & $<0.0001$
\end{tabular}

ACV: accidente cerebrovascular isquémico; AIT: accidente isquémico transitorio; EAR: estenosis de arteria renal. HR: hazard ratio. IC: intervalo de confianza. Adaptado de Kalra, et al. ${ }^{16}$

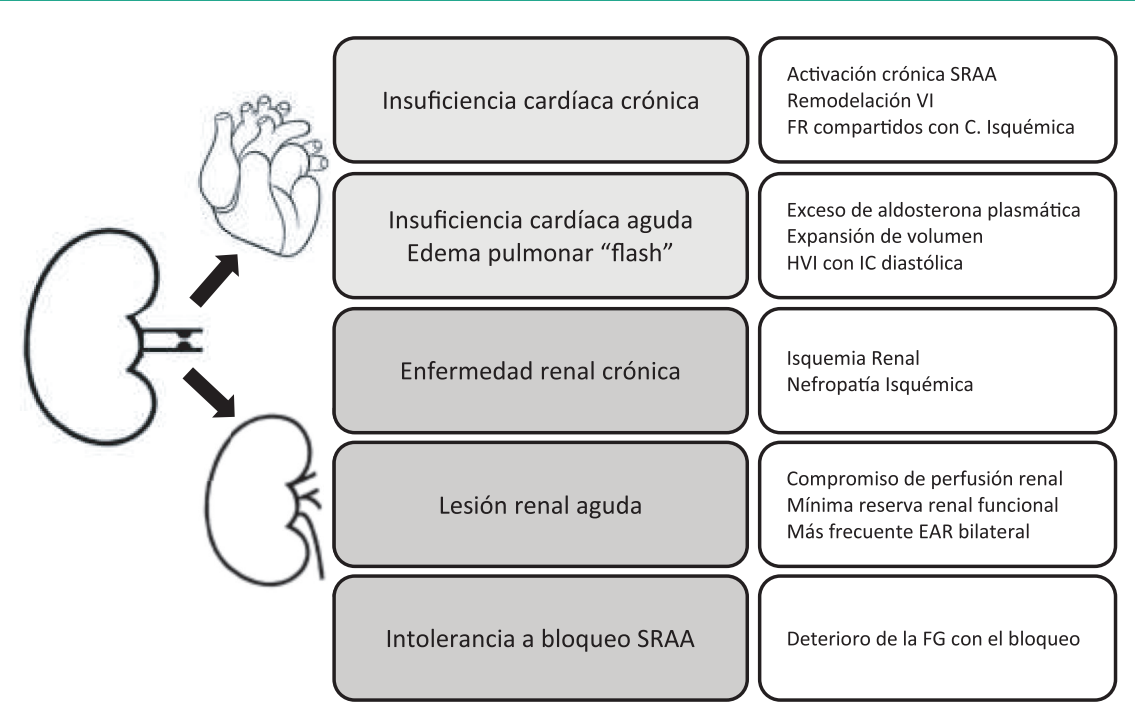

Figura 3. Manifestaciones clínicas de la EAR más allá de la HTRV. EAR: estenosis de la arteria renal; FG: filtración glomerular; FR: factores de riesgo; IC: insuficiencia cardíaca; HTRV: hipertensión renovascular; HVI: hipertrofia ventricular izquierda; SRRA: sistema renina-angiotensina-aldosterona; VI: ventrículo izquierdo.

eran mujeres. La edad promedio al diagnóstico fue de 45.3 años y mostró un amplio predomino de raza blanca (48 pacientes $)^{27}$. El registro norteamericano de DFM fue pionero en el plano mundial (inició en 2008). En 2012 registraba una población de 447 pacientes ingresados, con una edad media al diagnóstico de 51.9 años y una edad media al diagnóstico de HTA de esa misma población de 43 años. Del total de pacientes, 95\% era de raza blanca y $91 \%$ correspondía a mujeres ${ }^{28}$. El registro franco-belga (ARCADIA) comunicó un total de 469 pacientes incluidos, una edad media al diagnóstico de 53 años, $84 \%$ de mujeres, $88 \%$ de raza blanca y $91 \%$ con DFM de tipo multifocal ${ }^{29}$. Por último, el registro europeo-internacional (FEIRI), con más de 1,000 pacientes, que incluye los registros francés (NOMADE) y polaco (ARCADIA-POL), confirma un predominio de mujeres 
Tabla 3. Clasificación histológica y fenotipo arteriográfico de la DFM

\begin{tabular}{|c|c|c|c|}
\hline Tipo & Frecuencia (\%) & Histología & Aspecto angiográfico \\
\hline Media & $85 \%-100 \%$ & & \\
\hline DFM medial & Frecuente & Crestas de colágeno/pérdida de membrana elástica & $\begin{array}{l}\text { "Cadena de perlas". Diámetro del } \\
\text { grano más grande que la luz }\end{array}$ \\
\hline DFM perimedial & Rara & Hiperplasia del músculo liso & $\begin{array}{l}\text { "Cadena de perlas". Diámetro del } \\
\text { grano más pequeño que la luz }\end{array}$ \\
\hline Hiperplasia medial & Muy rara & & Estenosis lisa sin lecho vascular \\
\hline Íntima & $<10$ & $\begin{array}{l}\text { Depósito circunferencial de colágeno en la íntima; } \\
\text { fragmentación o duplicación de la elástica interna }\end{array}$ & Estenosis lisa elongada y concéntrica \\
\hline Adventicia & $<1$ & $\begin{array}{l}\text { Reemplazo de la adventicia y tejido subyacente por } \\
\text { tejido colágeno fibroso denso }\end{array}$ & $\begin{array}{l}\text { Estenosis lisa o atenuación difusa de } \\
\text { la luz vascular }\end{array}$ \\
\hline
\end{tabular}

DFM: displasia fibromuscular.

Adapatado de Vuong, et al. ${ }^{26}$

Tabla 4. Distribución de las lesiones de DFM de acuerdo con el territorio vascular

\begin{tabular}{|l|c|c|c|}
\hline Lecho vascular & DFMc (n) & DFMs (n) & $\%$ \\
\hline Arteria renal & 294 & 369 & 79.7 \\
\hline Carótida extracraneal & 251 & 338 & 74.3 \\
\hline Carótida intracraneal & 35 & 206 & 17 \\
\hline Arteria vertebral & 82 & 224 & 36.6 \\
\hline Arterias mesentéricas & 52 & 198 & 26.3 \\
\hline Arterias MMII & 42 & 70 & 60 \\
\hline Arterias MMSS & 10 & 63 & 15.9 \\
\hline Aorta & 0 & 145 & 0 \\
\hline
\end{tabular}

DFMc: displasia fibromuscular confirmada, número de pacientes con DFM confirmada en ese territorio. DFMs: displasia fibromuscular sospechada, número de pacientes en los que se realizó estudio radiológico en busca de DFM:

$\%$ : porcentaje de pacientes, los enfermos estudiados en los que se confirmó DFM. Adaptado de United States Registry for Fibromuscular Dysplasia ${ }^{28}$.

(82\%) y caucásicos $(88 \%)^{30}$. La edad promedio al diagnóstico fue de 46 años. De forma adicional, este registro describe $72 \%$ de DFM multifocal y $57 \%$ de DFM con lesiones en más de un territorio vascular (multivaso) ${ }^{30}$.

Si bien se han propuesto diversos factores genéticos, mecánicos y hormonales en la génesis de la DFM, la causa se conoce poco. Se han descrito casos de DFM familiar con un patrón de herencia autosómico dominante, así como casos esporádicos ${ }^{25}$. En los registros de DFM que integran un elevado número de casos sólo una minoría de pacientes corresponde a casos familiares ${ }^{25}$. Es por ello que no se aconseja el estudio genético sistemático en

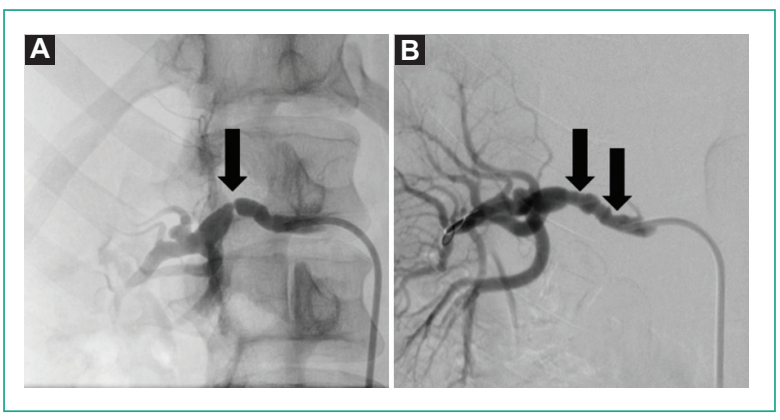

Figura 4. Arteriografía de la arteria renal. A: displasia fibromuscular focal; se observa una estenosis única con dilatación posestenótica. B: displasia fibromuscular multifocal; se observan diferentes áreas de estenosis que alternan con otras de dilatación de la arteria y el aspecto en "cadena de cuentas" (imágenes aportadas por el autor).

pacientes con DFM ${ }^{25}$. Dentro de los factores ambientales, el tabaquismo ha mostrado una relación con los casos de DFM renal. Otros factores como la exposición endógena 0 exógena a hormonas femeninas también se han vinculado con el desarrollo de la enfermedad, pero el vínculo definitivo permanece aún sin comprenderse ${ }^{25}$.

Las arteritis inflamatorias son una causa muy inhabitual de HTRV. En este grupo se incluyen las vasculitis de mediano y gran vaso: Takayasu, arteritis de células gigantes, enfermedad de Behçet y enfermedad de Kawasaki, entre otras. En la figura 5 se esquematizan las posibles causas de estenosis de arteria renal. 


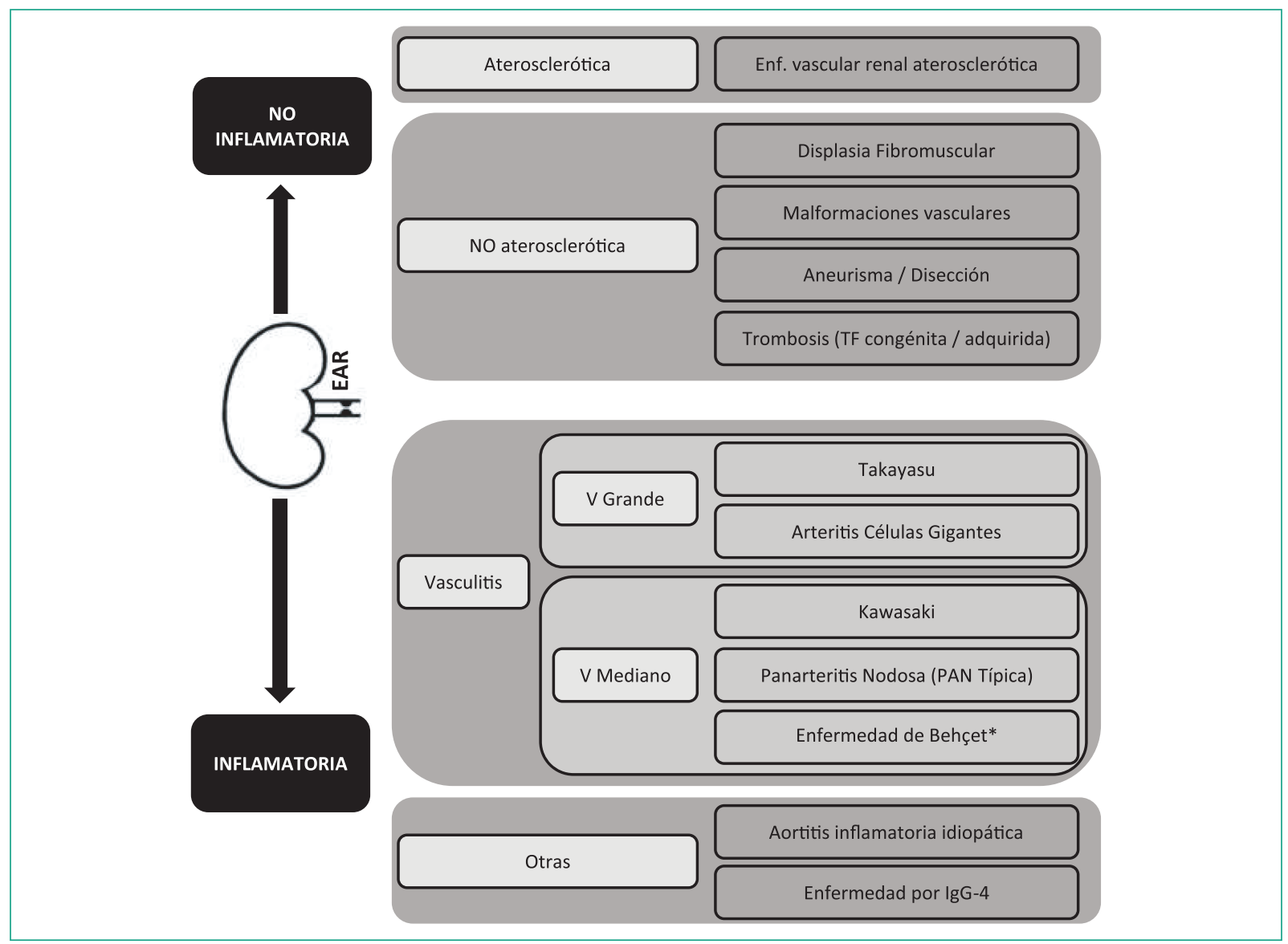

Figura 5. Causas de EAR. EAR: estenosis de arteria renal; IgG4: enfermedad relacionada con inmunoglobulina $\mathrm{G}$ subtipo 4; PAN: panarteritis nodosa; TF: trombofilia. V grande: vasculitis de vaso grande; $V$ mediano: vasculitis de vaso mediano. *En la enfermedad de Behçet son más frecuentes los aneurismas arteriales que las estenosis.

\section{¿En quién buscar hipertensión renovascular?}

No está indicada la búsqueda de EAR en pacientes asintomáticos. En enfermos en quienes se sospecha HTRV de causa aterosclerótica (situación más frecuente de la práctica clínica), se sugiere la búsqueda de EAR en los casos en que la evolución de la enfermedad y el área del paciente hagan necesaria y posible la revascularización en caso de hallarse. En la tabla 5 se muestran las situaciones clínicas que sugieren HTRV.

\section{¿Cómo estudiar la hipertensión renovascular?}

Existen múltiples herramientas para establecer el diagnóstico de EAR. Por lo general se ha considerado que la arteriografía renal es la norma de referencia contra la que se comparan todos los estudios de imagen, ya que ofrece alta sensibilidad y especificidad diagnóstica. No obstante, se trata de un estudio invasivo no exento de riesgos. Otros estudios invasivos de escaso uso en el medio son el ultrasonido intraarterial y la medición de presiones a ambos lados de la lesión (gradiente de presión). En la actualidad se emplean más los métodos no invasivos, entre los que destaca la ecografía Doppler, la angiotomografía y la angiorresonancia. Múltiples trabajos consideran que la angiotomografía es hoy día el estudio de referencia para las arterias renales. La arteriografía renal se reserva para los casos en que los estudios invasivos no son diagnósticos ${ }^{31}$ y en sujetos con diagnóstico confirmado en los que se plantea un tratamiento intervencionista.

La ecografía Doppler de arterias renales (EDAR) se recomienda como tamizaje en pacientes con sospecha de EAR (en arteria nativa y en el trasplante renal) y para el seguimiento de pacientes sometidos a 
Tabla 5. Situaciones clínicas que sugieren HTRV-EAR Inicio de la HTA antes de los 30 años

HTA grave que inicia luego de los 55 años y se relaciona con ERC o IC

HTA acompañada de soplo abdominal en el estudio clínico

Descontrol rápido y persistente de cifras tensionales en pacientes con HTA previamente controlada

HTA resistente (HTA no controlada a pesar de los antihipertensivos de cuatro clases diferentes, incluidos un diurético y un antagonista del receptor de mineralocorticoides, en dosis apropiadas)

Crisis hipertensiva (HTA no controlada junto con lesión renal aguda, insuficiencia cardíaca aguda, encefalopatía hipertensiva o retinopatía hipertensiva de grados 3-4)

Desarrollo de hiperazoemia o deterioro de la función renal luego del inicio de bloqueadores del SRAA

Atrofia renal inexplicada, 0 asimetría renal, 0 insuficiencia renal de causa inexplicada

Edema pulmonar flash

EAR: estenosis de la arteria renal; ERC: enfermedad renal crónica; HTA: hipertensión arterial; HTRV: hipertensión renovascular; IC: insuficiencia cardíaca; SRAA: sistema renina-angiotensina-aldosterona.

Tomado de Rooke, et al..$^{49}$

revascularización ${ }^{32}$. No se aconseja su uso en individuos asintomáticos, ya que su rendimiento diagnóstico no es adecuado ${ }^{33}$. Sus principales ventajas son su amplia disponibilidad y reproducibilidad; además, no emite radiaciones ionizantes ni usa medios de contraste intravenosos e identifica con adecuada precisión estenosis > 60\% (si bien sobreestima con frecuencia el grado estenótico). Entre sus desventajas figuran la necesidad de contar con experiencia técnica para su realización, el insumo de tiempos prolongados para valorar la arteria renal (el estudio bilateral completo puede tomar hasta 90 minutos), no permite determinar con precisión el grado (porcentaje) de estenosis, algunas áreas de la arteria renal son en particular laboriosas (el sector medio y en ocasiones el sector distal), tiene dificultades para identificar arterias renales accesorias, no valora en el mismo estudio otros territorios vasculares, no es tan precisa para valorar una afección diferente de la aterosclerótica e implica dificultades técnicas en individuos obesos y ante gas intestinal ${ }^{34}$. En un acuerdo entre múltiples sociedades científicas estadounidenses ${ }^{33}$ se especificaron requerimientos mínimos que debe cumplir un estudio EDAR (tabla 6). Múltiples estudios han validado el uso de EDAR para el diagnóstico de EAR en
Tabla 6. Requerimientos mínimos que debe cumplir una ecografía Doppler de arterias renales ${ }^{33}$

Deben documentarse imágenes en escala de grises o Doppler a color que deben incluir al menos:

- Aorta adyacente a la emergencia de las arterias renales

- Arterias renales

- Venas renales

- Imagen en escala de grises de riñones con medida de polo a polo renal bilateral

Debe documentarse Doppler espectral y medidas de velocidad que deben incluir como mínimo:

- Aorta adyacente a las arterias renales

- Arteria renal proximal, media y distal

- Arterias hiliares e intraparenquimatosas

- Arterias renales accesorias, si están presentes

- Venas renales (no se requiere medir velocidades)

Tomado de Mohler, et al..$^{33}$

comparación con la angiografía (norma de referencia). La mayoría encuentra sensibilidad y especificidad diagnósticas adecuadas, ambas $>80 \%^{34}$. Se trata de un estudio inocuo, ampliamente disponible y apropiado para valorar la EAR cuando existe esta presunción diagnóstica. Constituye la herramienta imagenológica inicial en la valoración de la EAR. Deben utilizarse de forma complementaria otros métodos de imagen para confirmar los hallazgos antes de definir una conducta terapéutica invasiva ${ }^{31}$.

La angiotomografía (angio-TC) de arterias renales ha sustituido en la última década a la arteriografía renal en el diagnóstico de EAR. Esto se debe por un lado al gran avance tecnológico que ha permitido desarrollar equipos con múltiples detectores que obtienen imágenes de muy alta resolución en muy cortos períodos de tiempo (escasos segundos) y, por otro lado, a un mayor conocimiento de la enfermedad de los médicos radiólogos que ha acompañado al desarrollo tecnológico.

Además del diagnóstico nosológico, la angio-TC permite definir o aproximarse a un diagnóstico etiológico: EAR aterosclerótica, DFM, disección, vasculitis, aneurismas, entre otras. Para el estudio de la HTRV se requiere la administración de medio de contraste yodado por vía intravenosa (habitualmente un bolo de 100-125 ml). La región a estudiar debe extenderse desde las cúpulas diafragmáticas hasta el sector distal de los ejes arteriales ilíacos, a efectos de identificar arterias renales accesorias de nacimiento atípico, así como afectación propia de la aorta abdominal que no se observaría si la adquisición se centra de forma exclusiva en el sitio donde típicamente emergen las arterias renales ${ }^{35}$. Algunos patrones lesivos identificados 
por la angio-TC se relacionan con las diferentes causas de EAR. En la enfermedad aterosclerótica, la estenosis compromete casi siempre el ostium y el área proximal de la arteria renal (primeros 10-20 mm). Puede visualizar además trombos murales, calcificación, placas ulceradas y dilatación posestenótica. Ha demostrado una sensibilidad de $64 \%$ a $100 \%$ y una especificidad de $94 \%$ a $99 \%$ para el diagnóstico de EAR aterosclerótica $^{31,36}$ (Fig. 6). Con los avances en la calidad y precisión de los equipos ha mejorado en grado significativo el diagnóstico y la clasificación de la DFM. La angio-TC identifica con frecuencia la DFM multifocal con la morfología en "cadena de cuentas", así como lesiones focales. A diferencia de la enfermedad aterosclerótica, la DFM se ubica las más de las veces en el sector medio o distal de la arteria renal. Hasta en el $60 \%$ de los casos pueden identificarse lesiones bilaterales. Asimismo, la angio-TC es capaz de reconocer complicaciones como disección, oclusión o trombosis vinculadas con la DFM ${ }^{35}$.

$\mathrm{La}$ angiorresonancia (angio-RM) de arterias renales es un estudio que obtiene imágenes adecuadas de las arterias renales. Puede realizarse con contraste por vía intravenosa (gadolinio), lo que representa la situación ideal, o sin el uso de contraste mediante secuencias angiográficas como el TOF (time of flight) en los pacientes con filtrado glomerular disminuido y riesgo de fibrosis nefrogénica sistémica por gadolinio, pero con obtención de imágenes con menor precisión diagnóstica. A diferencia de la angio-TC, no es capaz de visualizar el calcio de las placas de ateroma, lo que es una desventaja en términos de delinear con claridad la anatomía de vaso y una ventaja en tanto que posibilita una clara visualización de la luz arterial sin las distorsiones de la imagen que el calcio propicia ${ }^{37}$. Se considera una alternativa segura a la angio-TC en pacientes con deterioro de la filtración glomerular o alergia al medio de contraste yodado. En algunos estudios en los que se comparó con la arteriografía convencional se reconoció que la angio-RM tiende a sobreestimar el grado de estenosis; realizada con gadolinio tiene una sensibilidad de $97 \%$ y una especificidad de $85 \%$ para el diagnóstico de EAR aterosclerótica ${ }^{38}$. Hay pocos datos en las publicaciones médicas que valoren el desempeño de la angio-RM en el diagnóstico de la DFM, pero se considera que es inferior que en la enfermedad aterosclerótica ${ }^{37}$.

En la figura 7 se esquematiza un posible algoritmo de estudio. En todos los casos es fundamental la participación del radiólogo, sea al evaluar las imágenes

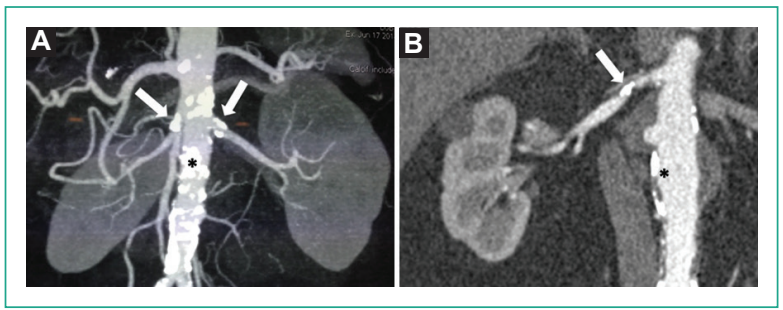

Figura 6. Angiotomografía de arterias renales. A: estenosis bilateral, mayor a la derecha con asimetría renal. B: estenosis unilateral del riñón derecho. ${ }^{*}$ Aorta patológica, con paredes irregulares y placas de ateroma calcificadas (imágenes aportadas por el autor).

para aproximarse a un diagnóstico o al asesorar al médico tratante respecto del algoritmo diagnóstico.

\section{Tratamiento}

Al igual que en otras anomalías (pero en especial en ésta), el tratamiento debe individualizarse. Deben tenerse en cuenta al definir la mejor conducta terapéutica la opción del paciente, la edad, las comorbilidades (habituales), el control de cifras tensionales con el tratamiento médico, la enfermedad renal y su progresión, el pronóstico funcional renal y la expectativa de vida, entre otros factores. Se describen a continuación las opciones terapéuticas en la HTRV de causa aterosclerótica y la secundaria a DFM. No se describe el tratamiento de otras causas relacionadas con HTRV en virtud de su baja frecuencia y los límites de este capítulo.

\section{Displasia fibromuscular}

El tratamiento médico es el antiplaquetario. No se dispone de adecuados estudios que valoren la utilidad de la antiagregación en este grupo de pacientes. En sujetos con episodios trombóticos o tromboembólicos, en ausencia de disección o aneurismas, u otras contraindicaciones, parece razonable la antiagregación plaquetaria (ácido acetilsalicílico a dosis de 75-100 mg VO al día), tanto en pacientes sintomáticos como en asintomáticos ${ }^{25}$.

Para el tratamiento de la HTA, el valor objetivo de las cifras tensionales no difiere del de la población general, por lo que se recomienda mantener el mismo objetivo terapéutico. Es aconsejable para ello el tratamiento con inhibidores de la enzima convertidora de angiotensina (IECA) o antagonistas de los 


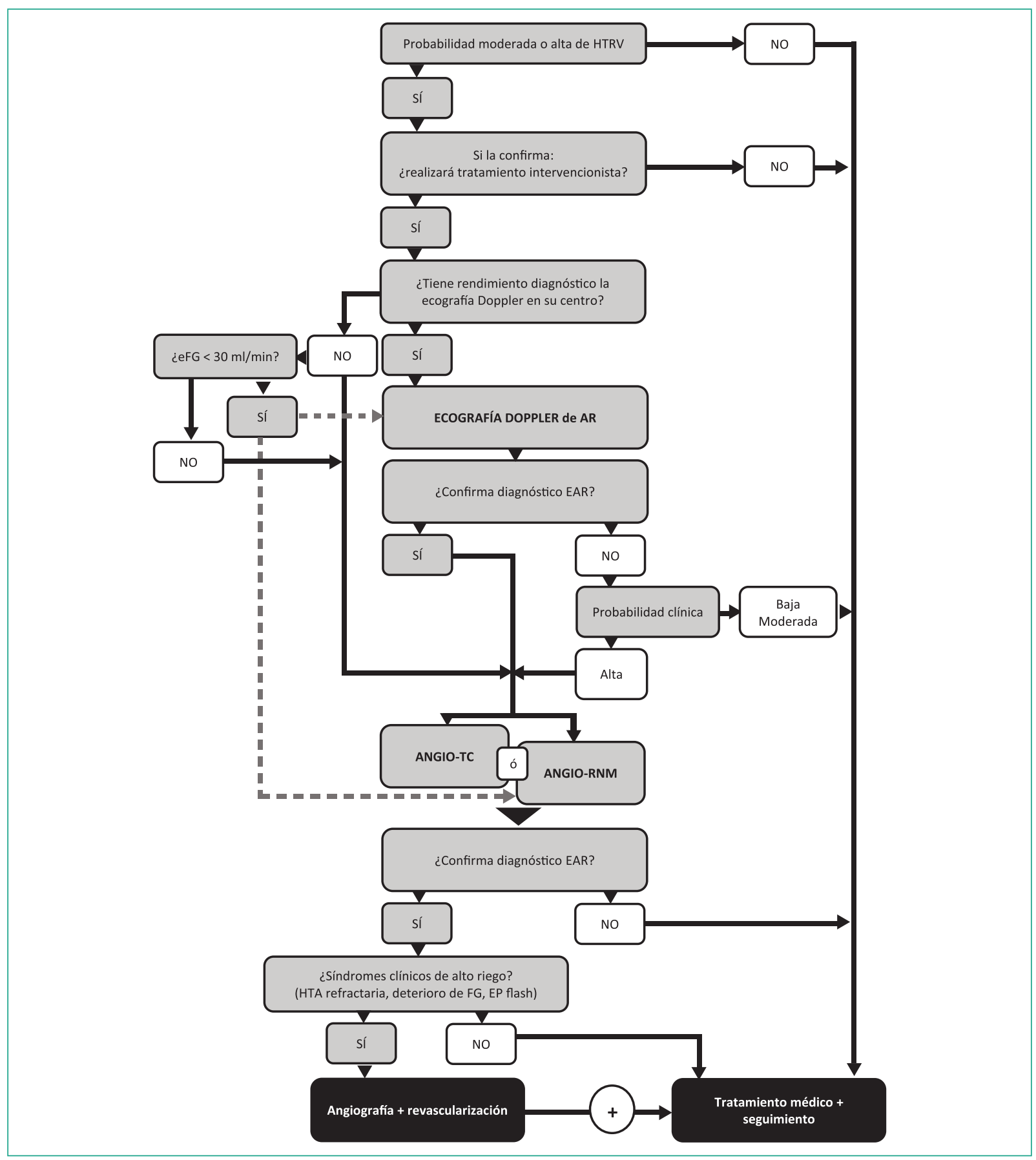

Figura 7. Algoritmo de estudio de HTRV. HTRV: hipertensión renovascular; EAR: estenosis de la arteria renal; eFG: filtración glomerular calculada. AR: arteria renal; angio-TC: angiotomografía; angio-RNM: angiorresonancia; EP: edema pulmonar.

receptores de angiotensina 2 (ARA-2) ${ }^{25}$. A diferencia de la enfermedad vascular aterosclerótica, la administración sistemática de estatinas no está indicada en esta población, y sólo deben prescribirse en función de los mismos objetivos terapéuticos adecuados a valores del perfil de lípidos plasmáticos, edad y factores de riesgo vascular. En todos los casos se debe recomendar la cesación del tabaquismo. La persistencia del hábito tabáquico se ha vinculado con mayor desarrollo de aneurismas y más episodios adversos e incluso mayor necesidad de procedimientos vasculares ${ }^{25}$. 
Entre los tratamiento intervencionistas en los pacientes con evidencia radiológica de DFM, la angiografía es todavía la norma de referencia del abordaje diagnóstico-terapéutico. Es importante, en función de la disponibilidad de cada centro, la medición de los gradientes de presión, con el objetivo de no realizar angioplastias innecesarias (en particular en la variante multifocal). En los pacientes con un gradiente de presión significativo está indicada la angioplastia. No se ha demostrado el beneficio de la colocación de stent en la DFM y en algunos estudios es un factor vinculado a la reestenosis, por lo que se reserva para la aparición de complicaciones. Se recomienda mantener la antiagregación con ácido acetilsalicílico (75-100 mg) por tiempo indefinido después de practicar la angioplastia ${ }^{25}$.

\section{Estenosis aterosclerótica}

Para las estenosis unilaterales ${ }^{31,33,39}$, todos los pacientes deben recibir tratamiento médico. En condiciones ideales, el tratamiento antihipertensivo debe incluir el bloqueo del sistema renina-angiotensina-aldosterona (inhibidor de la enzima convertidora de angiotensina o bloqueadores del receptor de angiotensina). Si no se consigue un control óptimo se recomienda añadir diuréticos de tipo tiazidas, bloqueadores de los canales de calcio, antagonistas de los receptores de mineralocorticoides o betabloqueadores.

Para el tratamiento intervencionista, los estudios controlados aleatorizados (ECA) no mostraron superioridad de la revascularización sobre el tratamiento médico en términos del control de cifras tensionales, preservación de la función renal o disminución de episodios cardiovasculares mayores ${ }^{40-42}$. En función de ello, la revascularización no está indicada en todos los pacientes. Se sugiere la revascularización mediante angioplastia percutánea (o en última instancia quirúrgica) en las siguientes situaciones ${ }^{24,31}$ :

- Corta evolución entre el diagnóstico de hipertensión arterial y el diagnóstico de estenosis significativa.

- Falla del tratamiento médico (hipertensión resistente) para lograr el control tensional óptimo.

- Intolerancia al tratamiento médico.

- Edema pulmonar flash recurrente o insuficiencia cardíaca resistente.

- Deterioro progresivo de la filtración glomerular (sobre todo en estenosis bilateral o estenosis en riñón único funcional) sin otra causa que la explique.

En el tratamiento médico de las estenosis bilateral o unilateral en riñón único funcional ${ }^{31,33,39}$, la estenosis bilateral determina habitualmente una activación sostenida del eje renina-angiotensina-aldosterona y ello una retención hidrosalina y expansión del volumen extracelular. La combinación ideal de fármacos para esta situación debe incluir el bloqueo del sistema renina-angiotensina-aldosterona (inhibidores de la enzima convertidora de angiotensina o bloqueador del receptor de angiotensina) junto con diuréticos. La mayor limitante de este tratamiento suele ser el desarrollo de lesión renal aguda hemodinámica (disminución de la perfusión renal e intraglomerular) vinculada con el bloqueo renina-angiotensina-aldosterona, que puede agravarse con la adición de diuréticos que induzcan reducción del volumen extracelular y mayor activación de angiotensina II. Esta caída de la filtración glomerular también puede ocurrir con menor frecuencia al iniciar cualquier otro antihipertensivo, relacionado sobre todo con la caída de la perfusión renal. El riesgo de deterioro del filtrado glomerular no debe ser una contraindicación para el bloqueo renina-angiotensina-aldosterona en estos pacientes, sino un motivo para mantener una vigilancia estrecha una vez que se inician. En la mayoría de los pacientes, el descenso de la filtración glomerular es leve y el aumento de la creatininemia $>30 \%$ de los valores basales al inicio del bloqueo se observa en menos del $10 \%$ de los casos de estenosis bilateral. En algunos pacientes, este aumento de la creatininemia pueda atenuarse al suspender el tratamiento diurético. El deterioro de la filtración glomerular con el inicio del tratamiento médico, si no es grave, no modifica el pronóstico del paciente a largo plazo ${ }^{43}$.

Uno de los riesgos del tratamiento médico aislado en pacientes con estenosis bilateral (o unilateral con riñón único funcional) es que el descenso de la presión arterial cause nefropatía isquémica con atrofia progresiva del parénquima renal.

En cuanto al tratamiento intervencionista, las indicaciones de revascularización con angioplastia percutánea (o quirúrgica) son las mismas expuestas en el tratamiento de la estenosis unilateral. En este grupo de pacientes cobra más valor la indicación de revascularización cuando existe filtración glomerular deteriorada que no permite mantener un tratamiento antihipertensivo óptimo (aumento persistente de la creatininemia con el bloqueo del eje renina-angiotensina-aldosterona o con cualquier otro tratamiento antihipertensivo).

\section{Discusión}

Se revisaron diferentes aspectos relacionados con causas, conductas diagnósticas y tratamientos de la 
HTRV secundaria a EAR de origen aterosclerótico y displasia fibromuscular.

El aspecto central en la EAR es identificar su causa, ya que las decisiones terapéuticas y el pronóstico son muy diferentes. En la EAR por DFM, los aspectos diagnósticos y terapéuticos alcanzan un mayor grado de sistematización y consenso ${ }^{25}$.

Sin embargo, en la EAR por aterosclerosis, el aspecto más difícil es la selección de los pacientes que podrían beneficiarse del tratamiento intervencionista. Los estudios sobre los que se basa la mayor parte de recomendaciones ${ }^{24,31}$ encuentran beneficio sobre todo en los pacientes que tienen un riesgo clínico alto, 0 fenotipo de "alto riesgo". Cooper, et al. ${ }^{40}$ (estudio CORAL) valoraron a 947 pacientes con EAR aterosclerótica confirmada e HTA bajo tratamiento con dos o más fármacos o enfermedad renal crónica. Los individuos se aleatorizaron 1:1 en dos grupos: el primero recibió tratamiento médico para la HTA y el otro tratamiento médico junto con angioplastia de la AR con colocación de stent. Los criterios de valoración fueron la aparición de episodios cardiovasculares mayores: muerte de causa cardiovascular, insuficiencia renal progresiva y necesidad de tratamiento de reemplazo renal. En un seguimiento de 43 meses, el desarrollo de episodios (criterio de valoración primario) no fue diferente en el grupo que recibió tratamiento intervencionista en comparación con el que recibió sólo tratamiento médico (35.1\% vs. 35.8\%; IC95\%, 0.76-1,17; p = 0.58). Estos resultados permiten concluir que el tratamiento con angioplastia y colocación de stent no confiere beneficio adicional al tratamiento médico para prevenir episodios cardiovasculares y renales mayores ${ }^{40}$. El mismo grupo de investigadores ${ }^{44}$ intentó probar la hipótesis de que los gradientes de presión en la arteria renal, la gravedad de la estenosis o la gravedad de la HTA basal podían afectar los resultados finales luego de la colocación de stent en la arteria renal. Sin embargo, el tratamiento intervencionista tampoco mostró beneficio en estos subgrupos ${ }^{44}$. Wheatley, et al. ${ }^{42}$ (estudio ASTRAL) aleatorizaron a 806 pacientes para recibir tratamiento con angioplastia con stent de la arteria renal en relación con tratamiento médico o tratamiento médico solo. El criterio de valoración primario era la función renal calculada a partir de la creatininemia y los secundarios el valor de la presión arterial, desarrollo de episodios cardiovasculares mayores y muerte. Durante un periodo de cinco años, la tasa de progresión de la enfermedad renal fue menor en el grupo que recibió revascularización (IC95\%, -0.002 a 0.13; $p=0.06)$. No hubo diferencias significativas en los valores de presión arterial sistólica y los valores de presión arterial diastólica disminuyeron en el grupo que recibió revascularización. Sin embargo, no hubo diferencia en los efectos duros. La aparición de episodios renales (IC95\%, 0.67 a 1.4; $p=0.88$ ), cardiovasculares mayores (IC95\%, 0.75 a $1.19 ; p=0.61)$ y muerte (IC95\%, 0.69 a 1.18; $p=0.46$ ) fueron similares en ambos grupos $^{42}$. Bax, et al. ${ }^{41}$ (estudio STAR) valoraron la eficacia y seguridad del tratamiento con angioplastia con stent para mejorar la función renal de pacientes con EAR. Aleatorizaron a 140 pacientes con depuración de creatinina $<80 \mathrm{ml} / \mathrm{min}$ y $E A R \geq 50 \%$. Sesenta y cuatro pacientes recibieron tratamiento con stent junto con tratamiento médico y 76 pacientes sólo tratamiento médico. El criterio de valoración primario fue la mejoría de la función renal $\geq 20 \%$ y los secundarios seguridad, y morbilidad y mortalidad de causa cardiovascular. Alcanzaron el objetivo primario $16 \%$ de los pacientes tratados con stent contra $22 \%$ de los que recibieron sólo tratamiento médico. No se identificaron diferencias en los otros criterios de valoración secundarios valorados. Estos autores confirman que el tratamiento con angioplastia y stent no muestra claro beneficio en lo que refiere al avance de la enfermedad renal en pacientes con EAR ${ }^{41}$. Ritchie, et al. ${ }^{45}$ valoraron el beneficio de la revascularización percutánea en pacientes con EAR aterosclerótica y presentaciones clínicas de alto riesgo, incluidos edema pulmonar flash, deterioro rápido de la función renal e hipertensión arterial resistente. El estudio valoró en forma retrospectiva a 467 pacientes con $E A R \geq 50 \%$ tratados según la preferencia del médico tratante. Se presentaron con edema pulmonar flash $7.8 \%$, hipertensión arterial resistente $24.3 \%$ y deterioro rápido de la función renal $9.7 \%$, mientras que $49 \%$ de los pacientes no mostró ninguno de estos fenotipos de alto riesgo. En este estudio, la revascularización reveló una reducción del riesgo de muerte (HR 0.4; IC95\%, 0.2 a 0.9; $p=0.01$ ), pero no del riesgo de desarrollar episodios cardiovasculares 0 enfermedad renal extrema, en el grupo con edema pulmonar flash. No obstante, la revascularización no se acompañó de reducción de riesgo de ninguno de los criterios de valoración en los grupos con rápido deterioro de la función renal o hipertensión resistente ${ }^{45}$. Además, Van den Berg, et al. ${ }^{46}$ realizaron una revisión sistemática de la eficacia de la angioplastia renal en pacientes con edema pulmonar o insuficiencia cardíaca congestiva. Incluyeron 25 estudios, 79 pacientes con EAR y edema pulmonar flash o insuficiencia cardíaca y hallaron que $76 \%$ de los pacientes con edema flash no tiene recurrencia de éste luego de la 
angioplastia y que en los casos que hay recurrencia del edema pulmonar se debe a reestenosis de la arteria renal. En pacientes con insuficiencia cardíaca e insuficiencia renal, la gravedad de los síntomas mejoró luego de la angioplastia. Los autores concluyeron que la evidencia obtenida de los estudios justifica la recomendación del tratamiento intervencionista en pacientes con EAR aterosclerótica que muestran edema flash, insuficiencia cardíaca congestiva o insuficiencia renal ${ }^{46}$. Por último, un metaanálisis de Kumbhani, et al. ${ }^{47}$ analiza el beneficio de la revascularización percutánea en relación con tratamiento médico comparada con el tratamiento médico solo en pacientes con EAR aterosclerótica; se incluyó a seis estudios aleatorizados con un total de 1,208 pacientes. El resultado muestra una reducción del número de fármacos antihipertensivos (-0.26; IC 95\%, -0.39 a -0.13; $p<0.01)$ en el grupo sometido a revascularización en comparación con tratamiento médico solo. Sin embargo, no revela beneficio adicional sobre la evolución de la creatininemia y la aparición de episodios clínicos ${ }^{47}$. Con base en lo anterior, puede concluirse que, en la EAR de causa aterosclerótica, el beneficio del tratamiento intervencionista (angioplastia \pm colocación de stent), si es que existe, se limita a un grupo muy reducido y seleccionado de pacientes con un fenotipo de alto riesgo. Para tomar decisiones es necesaria la revisión "caso a caso" con el objetivo de indicar un tratamiento personalizado frente a cada situación.

\section{Conflicto de intereses}

Los autores declaran no tener conflicto de intereses.

\section{Bibliografía}

1. Boutari C, Georgianou E, Sachinidis A, Katsimardou A, Christou K, Piperidou $A$, et al. Renovascular hypertension: novel insights. Curr Hypertens Rev. 2019;15. doi:10.2174/1573402115666190416153321.

2. Dworkin LD, Cooper CJ. Renal-artery stenosis. N Engl J Med. 2009;20:1972-1978.

3. Fried JG, Morgan MA. Renal imaging: core curriculum 2019. Am J Kidney Dis. 2019;73:552-565.

4. Prince M, Tafur JD, White CJ. When and How should we revascularize patients with atherosclerotic renal artery stenosis? JACC Cardiovasc Interv. 2019;12:505-517.

5. Willis K, Cheung M, Slifer S. Abstract. Kidney Int Suppl. 2013;3:4.

6. Lerman L, Textor SC. Pathophysiology of ischemic nephropathy. Urol Clin North Am. 2001;28:793-803.

7. Goldblatt H, Lynch J, Hanzal RF, Summerville WW. Studies on experimental hypertension. J Exp Med. 1934;59:347-379.

8. Braun-Menéndez E, Fasciolo JC, Leloir LF, Muñoz JM. La sustancia hipertensora de la sangre del riñón isquemiado. Rev Soc Arg Biol. 1939;15:420-425.

9. Martínez-Maldonado M. Clinical conference pathophysiology of renovascular hypertension. Hypertension. 1991;17.

10. Lerman LO, Textor SC. Renal vascular disease. Springer. 2014.

11. Dieter R, Weber B. Renal artery stenosis: epidemiology and treatment. Int J Nephrol Renovasc Dis. 2014;169.

12. Hamilton G. Renovascular disease. Vasc Surg Princ Pract. 2017;39:589-602.
13. De Bruyne B, Manoharan G, Pijls NHJ, Verhamme K, Madaric J, Bartunek J, et al. Assessment of renal artery stenosis severity by pressure gradient measurements. J Am Coll Cardiol 2006;48:1851-1855.

14. Ritchie J, Kalra PA. Atherosclerotic renovascular disease: epidemiology and clinical manifestations. In: Lerman LO, Textor SC (eds). Renal vascular disease. Springer: Rochester, MN, 2014:3-21.

15. Hansen KJ, Edwards MS, Craven TE, Cherr GS, Jackson SA, Appel RG, et al. Prevalence of renovascular disease in the elderly: a population-based study. J Vasc Surg. 2002;36:443-451.

16. Kalra PA, Guo H, Kausz AT, Gilbertson DT, Liu J, Chen S-C, et al. Atherosclerotic renovascular disease in United States patients aged 67 years or older: risk factors, revascularization, and prognosis. Kidney Int. 2005;68:293-301.

17. Zierler R, Bergelin R, Davidson R, Cantwellcab K, Polissar N, Strandnessjr D. A Prospective study of disease progression in patients with atherosclerotic renal artery stenosis. Am J Hypertens. 1996;9:1055-1061.

18. Caps MT, Perissinotto C, Zierler RE, Polissar NL, Bergelin RO, Tullis MJ, et al. Prospective study of atherosclerotic disease progression in the renal artery. Circulation. 1998;98:2866-2872.

19. Satyapal KS, Haffejee AA, Singh B, Ramsaroop L, Robbs J V, Kalideen JM. Additional renal arteries incidence and morphometry. Surg Radiol Anat. 2001;23:33-38.

20. Gupta A, Tello R. Accessory renal arteries are not related to hypertension risk: a review of mr angiography data. Am J Roentgenol. 2004;182:1521-1524.

21. Glodny B, Cromme S, Wörtler K, Winde G. A possible explanation for the frequent concomitance of arterial hypertension and multiple renal arteries. Med Hypotheses. 2001;56:129-133.

22. Tash JA, Stock JA, Hanna MK. The role of partial nephrectomy in the treatment of pediatric renal hypertension. J Urol. 2003;169:625-8.

23. Bude RO, Forauer AR, Caoili EM, Nghiem HV. Is it necessary to study accessory arteries when screening the renal arteries for renovascular hypertension? Radiology. 2003;226:411-416.

24. Rooke TW, Hirsch AT, Misra S, Sidawy AN, Beckman JA, Findeiss LK, et al. 2011 ACCF/AHA focused update of the guideline for the management of patients with peripheral artery disease (Updating the 2005 Guideline). Circulation. 2011;124:2020-2045.

25. Gornik HL, Persu A, Adlam D, Aparicio LS, Azizi M, Boulanger M, et al. First International Consensus on the diagnosis and management of fibromuscular dysplasia. Vasc Med (United Kingdom). 2019;24:164-189.

26. Vuong P, Desoutter P, Mickley V, Bültmann B, Rothenberger-Janzen K, Guyot $\mathrm{H}$, et al. Fibromuscular dysplasia of the renal artery responsible for renovascular hypertension: a histological presentation based on a series of 102 patients. Vasa. 2004;33:13-18.

27. Aparicio LS, Alfie J, Barochiner J, Bluro I, Bresso P, Castellaro C, et al. Registro Argentino de Displasia Fibromuscular. Grupo de trabajo de Hipertensión Secundaria de la Sociedad Argentina de Hipertensión Arterial (SAHARA-DF). Bases y Resultados Preliminares. 2019;0:1.

28. Olin JW, Froehlich J, Gu X, Bacharach JM, Eagle K, Gray BH, et al. The United States Registry for Fibromuscular Dysplasia. Circulation. 2012;125:3182-3190.

29. Plouin PF, Baguet JP, Thony F, Ormezzano O, Azarine A, Silhol F, et al. High prevalence of multiple arterial bed lesions in patients with fibromuscular dysplasia: The ARCADIA Registry (Assessment of Renal and Cervical Artery Dysplasia). Hypertension. 2017;70:652-658.

30. Pappaccogli M, Di Monaco S, Warchoł-Celińska E, Lorthioir A, Amar L, Aparicio LS, et al. The European/International Fibromuscular Dysplasia Registry and Initiative (FEIRI)—clinical phenotypes and their predictors based on a cohort of 1000 patients. Cardiovasc Res. 2020. doi:10.1093/ cvr/cvaa102.

31. Aboyans V, Ricco JB, Bartelink M-LEL, Björck M, Brodmann M, Cohnert T, et al. 2017 ESC Guidelines on the Diagnosis and Treatment of Peripheral Arterial Diseases, in collaboration with the European Society for Vascular Surgery (ESVS). Eur Heart J. 2018;39:763-816.

32. Sciuto F, Langleib M. Eco Doppler en el diagnóstico de estenosis de la arteria renal. In: Mazzuchi N, Lacordelle F (eds). Hipertensión arterial renovascular y nefropatía isquémica. Oficina del Libro. Facultad de Medicina. Universidad de la República: Montevideo, 2000:55-61.

33. Mohler ER, Gornik HL, Gerhard-Herman M, Misra S, Olin JW, Zierler RE. ACCF/ACR/AIUM/ASE/ASN/ICAVL/SCAI/SCCT/SIR/SVM/SVS 2012 appropriate use criteria for peripheral vascular ultrasound and physiological testing part I: arterial ultrasound and physiological testing: a report of the American College of Cardiology Foundation a. J Am Coll Cardiol. 2012;60:242-276.

34. Weinberg I, Jaff MR. Renal artery duplex ultrasonography. In: Lerman LO, Textor SC (eds.). Renal vascular disease. Springer London: London, MN, 2014:211-229.

35. Vrtiska TJ. CT Angiography of the renal arteries. In: Lerman LO, Textor SC (eds). Renal vascular disease. Springer London: London, MN, 2014:231-246.

36. Rountas C, Vlychou M, Vassiou K, Liakopoulos V, Kapsalaki E, Koukoulis $G$, et al. Imaging modalities for renal artery stenosis in suspected renovascular hypertension: prospective intraindividual comparison of color Doppler US, CT angiography, GD-enhanced MR angiography, and digital substraction angiography. Ren Fail. 2007;29:295-302. 
37. Glockner JF. Renal MR angiography. In: Lerman LO, Textor SC (eds.) Renal vascular disease. Springer: Rochester, MN, 2014:247-258.

38. Tan KT, Van Beek EJR, Brown PWG, Van Delden OM, Tijssen J, Ramsay LE. Magnetic resonance angiography for the diagnosis of renal artery stenosis: a meta-analysis. Clin Radiol 2002;57:617-624.

39. Tafur JD, White CJ. Renal artery stenosis: when to revascularize in 2017 Curr Probl Cardiol. 2017; 42:110-135.

40. Cooper CJ, Murphy TP, Cutlip DE, Jamerson K, Henrich W, Reid DM, et al. Stenting and medical therapy for atherosclerotic renal-artery stenosis. N Engl J Med. 2014;370:13-22.

41. Bax L. Stent placement in patients with atherosclerotic renal artery stenosis and impaired renal function. Ann Intern Med. 2009;150:840.

42. ASTRAL Investigators W, Wheatley K, Ives N, Gray R, Kalra PA Moss JG, et al. Revascularization versus medical therapy for renal-artery stenosis. N Engl J Med. 2009;361:1953-62.

43. Cooper EL, Xie Y, Nguyen H, Brewster PS, Sholl H, Sharrett M, et al. Early rapid decline in kidney function in medically managed patients with atherosclerotic renal artery stenosis. J Am Heart Assoc. 2019;8.

44. Murphy TP, Cooper CJ, Matsumoto AH, Cutlip DE, Pencina KM, Jamerson K, et al. Renal artery stent outcomes. J Am Coll Cardiol. 2015;66:2487-2494.
45. Ritchie J, Green D, Chrysochou C, Chalmers N, Foley RN, Kalra PA. High-risk clinical presentations in atherosclerotic renovascular disease: prognosis and response to renal artery revascularization. Am J Kidney Dis. 2014;63:186-197.

46. van den Berg DTNA, Deinum J, Postma CT, van der Wilt GJ, Riksen NP. The efficacy of renal angioplasty in patients with renal artery stenosis and flash oedema or congestive heart failure: a systematic review. Eur J Heart Fail. 2012;14:773-781.

47. Kumbhani DJ, Bavry AA, Harvey JE, de Souza R, Scarpioni R, Bhatt DL, et al. Clinical outcomes after percutaneous revascularization versus medical management in patients with significant renal artery stenosis: a meta-analysis of randomized controlled trials. Am Heart. J 2011;161:622630.e1.

48. de Mast $Q$, Beutler JJ. The prevalence of atherosclerotic renal artery stenosis in risk groups: a systematic literature review. J Hypertens. 2009;27:1333-1340.

49. Rooke TW, Hirsch AT, Misra S, Sidawy AN, Beckman JA, Findeiss LK, et al. 2011 ACCF/AHA focused update of the guideline for the management of patients with peripheral artery disease (updating the 2005 guideline). Catheter Cardiovasc Interv. 2012;79:501-531. 